\title{
Stereoselective Radical Translocations
}

\author{
Philippe Renaud ${ }^{\star a}$, Florent Beaufils ${ }^{a}$, Fabrice Dénès $^{b}$, Laurence Feray ${ }^{c}$, Christoph Imboden ${ }^{a}$, and \\ Nikolai Kuznetsova
}

\begin{abstract}
The stereochemical outcome of intramolecular radical mediated hydrogen transfer (= radical translocation) is discussed. Low to excellent levels of stereocontrol are observed making such processes attractive for applications in target-oriented synthesis.
\end{abstract}

Keywords: H-Abstraction · C-H Activation · Asymmetric synthesis · Radicals · Stereochemistry

\section{Introduction}

Radical reactions are becoming a very valuable tool for organic synthesis. ${ }^{[1]}$ The mildness of the reaction conditions, their complementary nature to ionic processes, and the possibility of carrying out sequential reactions (cascade reactions) are some of the key factors of their success. Radical reactions can be used for highly stereoselective bond-forming reactions. The stereochemistry of radical cyclizations has been studied extensively and reliable stereoselectivity rules (the Beckwith-Schiesser-Houk model) have been proposed. ${ }^{[2]}$ Intramolecular hydrogen-atom abstraction (also called radical translocation) is a bond-breaking/bondforming process that is frequently encountered in radical reactions. ${ }^{[3]}$ It represents a unique mode for remote functionalization of unreactive $\mathrm{C}-\mathrm{H}$ bonds making it a very attractive synthetic transformation complementary to transition metal catalyzed processes. ${ }^{[4]}$ This short review article will

${ }^{\star}$ Correspondence: Prof. Dr. P. Renaud ${ }^{\mathrm{a}}$

Tel.: +41316314359

Fax: +41316313426

E-mail: philippe.renaud@ioc.unibe.ch

aUniversität Bern

Departement für Chemie und Biochemie

Freiestrasse 3

$\mathrm{CH}-3012$ Bern

bUniversité de Nantes

CEISAM UMR CNRS 6230

UFR des sciences et des techniques

2 , rue de la Houssinière

F-44322 Nantes Cedex 3, France

cUniversité Paul Cezanne Aix-Marseille III

Faculté des Sciences et Techniques

Avenue Escadrille Normandie Niemen

F-13397 Marseille Cedex 20, France

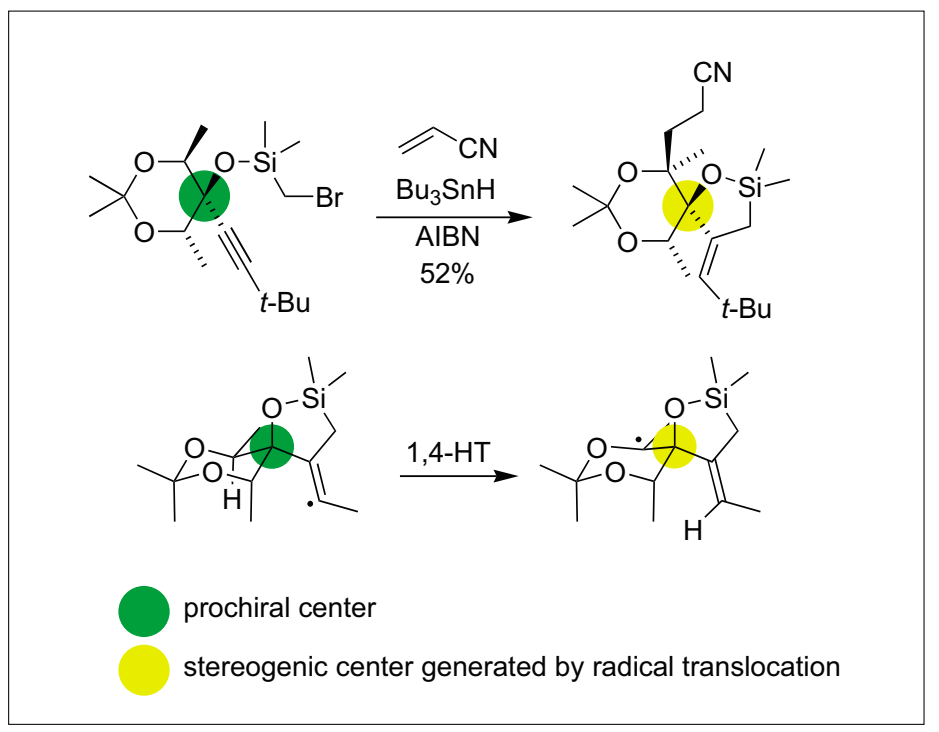

Scheme 1.

concentrate on the stereochemical aspect of radical translocation and more precisely on examples where the hydrogen transfer generates at least one new stereogenic center by selective activation of diastereotopic groups. Oxidation processes involving radical mediated $\mathrm{C}-\mathrm{H}$ bond activation have already been reviewed and will not be discussed here. ${ }^{[5]}$ Reactions have been classified according to the type of transformation and to mechanistic considerations.

\section{Radical Translocation - Intermolecular Radical Reaction}

Malacria and coworkers observed an unusual 1,4-hydrogen transfer during the radical cyclization of a propargylic bromomethyldimethylsilyl ether followed by trapping with acrylonitrile (Scheme 1). ${ }^{[6]}$ Interestingly, a prochiral center (green) is converted into a new stereogenic center (yellow) during the radical translocation. The stereochemistry of the H-transfer is fixed by the cyclic nature of the substrate. The consecutive trapping of the resulting anomeric radical is highly diastereoselective.

Cekovic and coworkers developed an efficient free radical phenylthio group transfer reaction by photolysis of benzenesulfenates. ${ }^{[7]}$ This process involves the formation of an alkoxyl radical, followed by a 1,5-hydrogen transfer, and final trapping of the alkyl radical by a $\mathrm{S}_{\mathrm{H}} 2$ reaction at the sulfur center of the starting sulfenate. Reaction of a gem-dimethyl substituted cyclohexane derivative has been reported to afford the rearranged sulfide in $60 \%$ yield as a single diastereomer (Scheme 2, reaction 1). In this process, the translocation process generates a new stereogenic center with complete stereochemical control due to the cyclic nature of the substrate. A similar result was obtained starting from the corresponding cyclohexyl nitrite (Scheme 2 , reaction 2 ) in the presence of acrylo- 


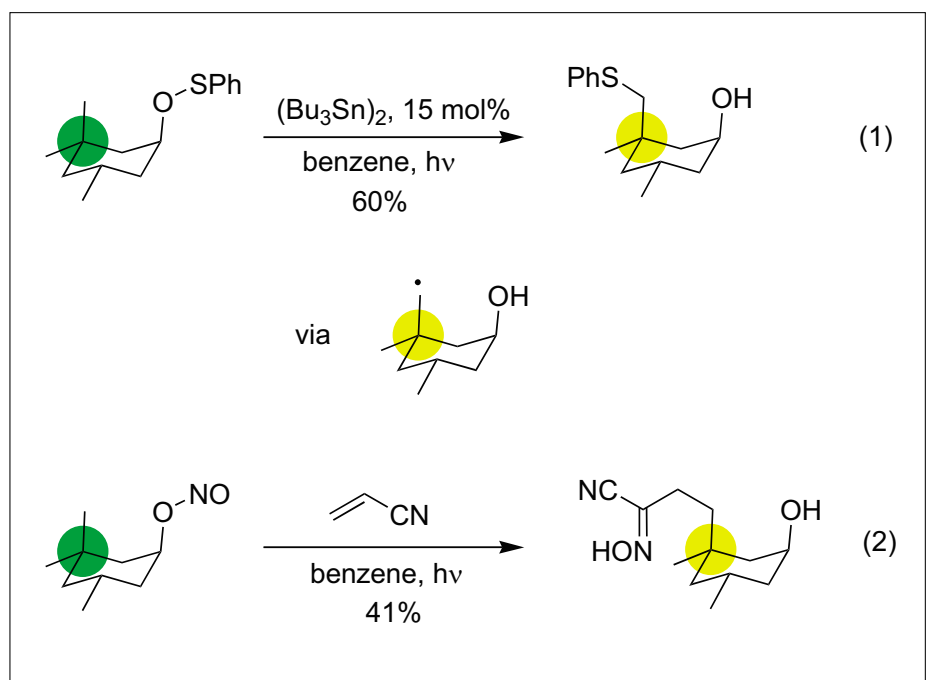

Scheme 2.

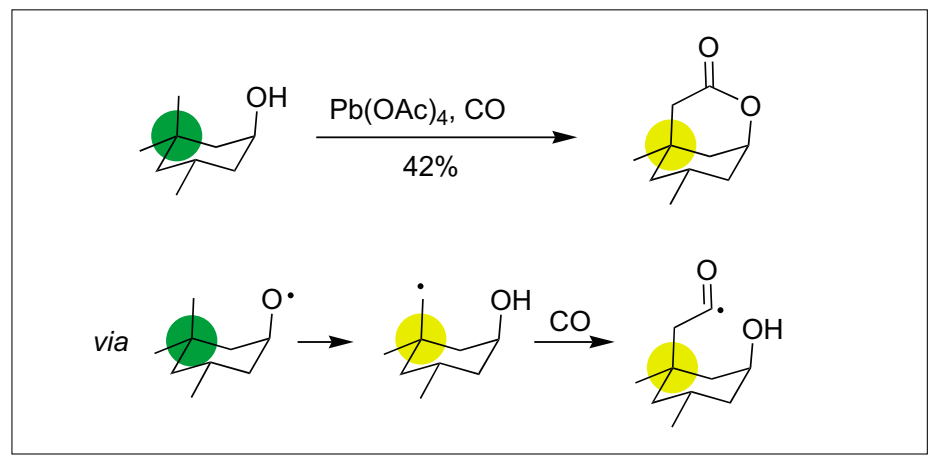

Scheme 3.

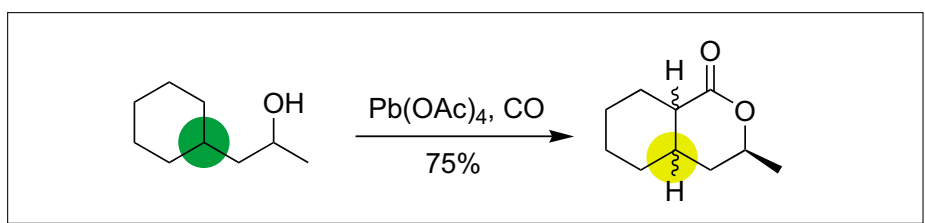

Scheme 4.

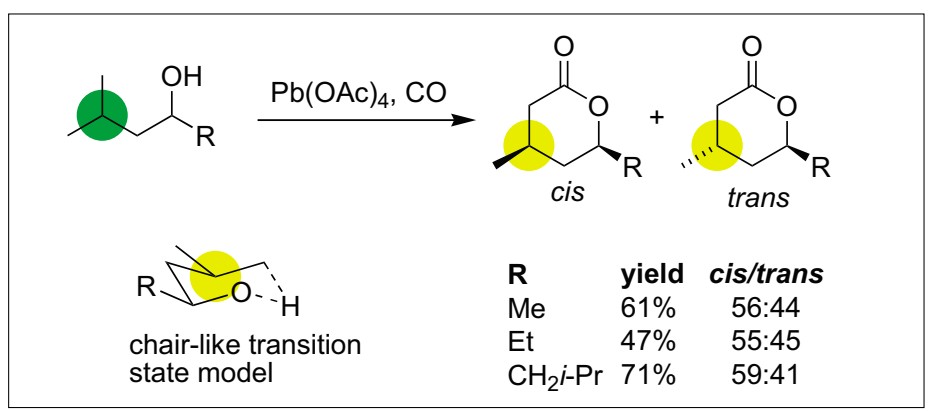

Scheme 5

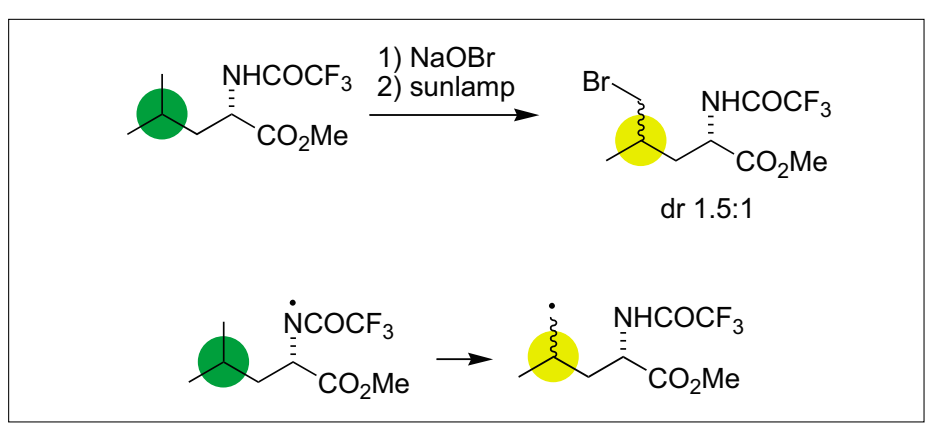

Scheme 6. nitrile. ${ }^{[8]}$ In this example, the translocated radical adds to acrylonitrile before transfer of the nitroso group.

Ryu developed a method for the preparation of $\delta$-lactones based on remote carbonylation of alcohols. ${ }^{[9]}$ This elegant procedure has been applied to several systems involving a stereoselective radical translocation. For instance, 3,3,5-trimethylcyclohexanol affords the bicyclic lactone as a single diastereomer (Scheme 3). ${ }^{[10]}$

Attempts to control the stereoselectivity in acyclic systems have been reported. For instance, 1-cyclohexyl-2-propanol gives the expected bicyclic lactone as a closed to equimolar mixture of four diastereomers demonstrating that neither the H-transfer nor the carbonylation steps are stereoselective (Scheme 4).

A low level of sterocontrol (up to 59:41) was reported when primary alkyl radicals were generated (Scheme 5). In this case, the low preference for the formation of cis-disubstituted $\delta$-lactones is best explained by a chair-like transition state model where both substituents are in equatorial position.

Recently, the Corey group has investigated free radical chain bromination of the Hofmann-Löffler-Freitag type starting from isoleucine and other $\alpha$-amino acids. ${ }^{[11]}$
In the case of $N$-trifluoroacetylleucine methyl ester (Scheme 6), the bromination reaction affords the primary bromide with a modest level of stereocontrol (diastereomeric ratio $d r=1.5: 1$ ). The relative configuration of the major product has not been determined.

\section{Radical Translocation - Radical Cyclization}

Malacria and coworkers reported the first example of a translocation-cyclization process of a vinyl radical involving a stereoselective 1,5-hydrogen transfer (Scheme 7). ${ }^{[12]}$ In this example, a 5-exo-dig cyclization of a bromomethylsilyl propargyl ether affords a vinyl radical that abstracts stereoselectively a hydrogen atom from one of the two diastereotopic methyl groups of the isopropyl substituent. After treatment of the crude product with methyllithium, a cyclopentanol is obtained in $74 \%$ yield as a single diastereomer. Interestingly, no product resulting from the 1,5-hydrogen transfer from the activated acetal $\mathrm{C}-\mathrm{H}$ bond is observed. In this transformation, two stereogenic centers are created during the H-transfer step; the two last centers result from the final cyclization step. A simple model based on steric hindrance explains the regioselectivity and the stereochemical outcome of the radical translocation.

Attempts to control the absolute stereochemistry of the hydrogen atom transfer step by use of chiral auxiliaries have been reported. ${ }^{[12]}$ An optically pure alkynyl sulfoxide affords the product resulting from a translocation-cyclization-fragmentation process in low yield (Scheme 8, reaction $3)$. Moreover, the product is racemic resulting from a lack of facial selectivity during the hydrogen abstraction process. A second attempt with a chiral phosphine oxide gave a low level of stereocontrol (Scheme 8, reaction 4).

We have investigated in depth the diastereoselectivity of the hydrogen transfer during the translocation step involving chiral acetals. ${ }^{[13]}$ The abstraction proceeds with good stereochemical control and is easily rationalized by a chair-like transition state in which the 2-methoxy group occupies an axial position as a result of the anomeric effect, and the non-reacting group at $\mathrm{C}(5)$ lies in a pseudoequatorial position (Scheme 9). This model is closely related to the one reported for the cyclization of chiral acetals. ${ }^{[14]}$ 


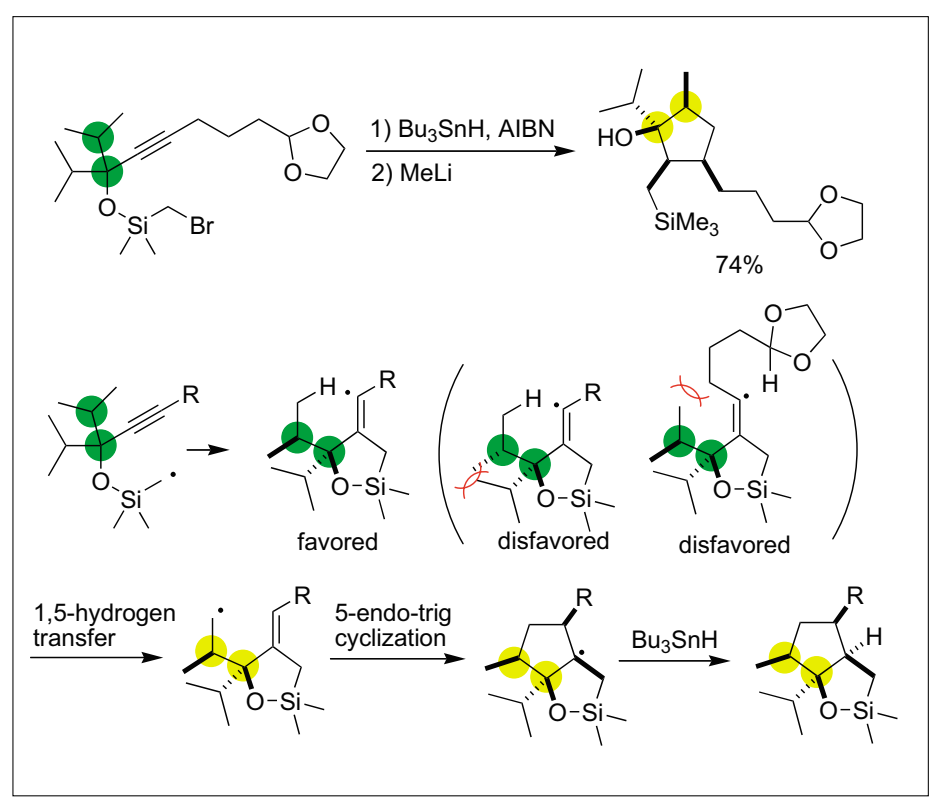

Scheme 7.
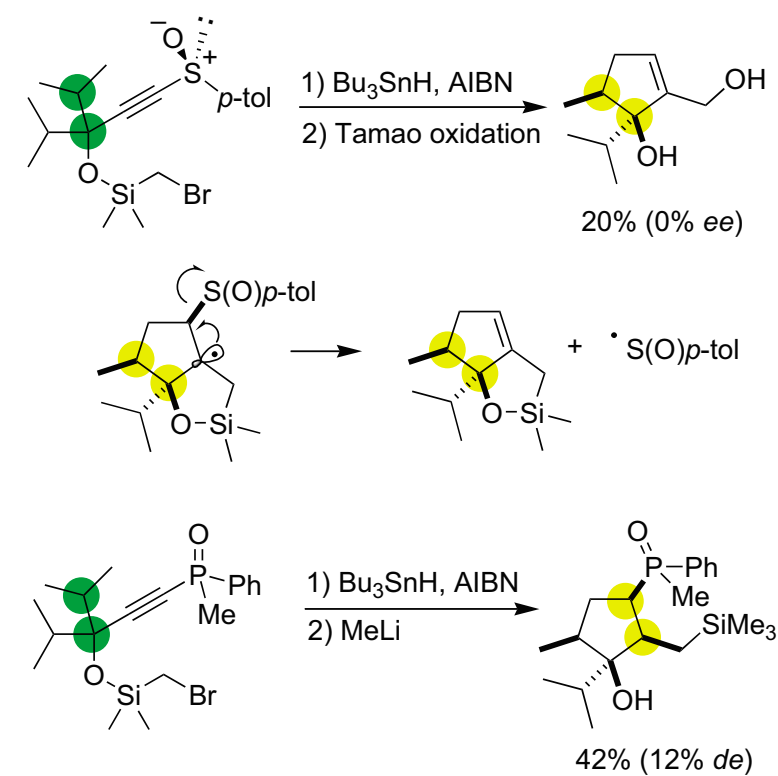

Scheme 8

To illustrate the synthetic potential of this process, the preparation of a enantiomerically enriched $\alpha$-methylenelactone using a diastereoselective hydrogen atom abstraction was performed (Scheme 10). ${ }^{[13]}$ For this purpose, the optically enriched propynal acetal $(96 \%$ ee) was isolated by HPLC on a chiral stationary-phase column. Reaction of the acetal under the thiophenol method followed by oxidation of the cyclic acetal with an excess of Jones reagent and treatment with 1,8-diazabicycloundec7-ene (DBU) affords the diastereomerically pure $\alpha$-methylene lactone with $88 \%$

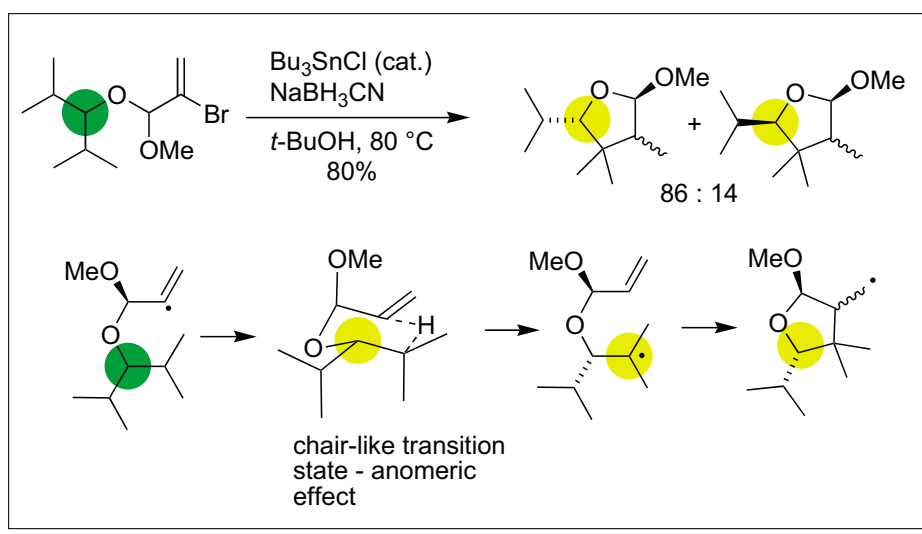

Scheme 9.

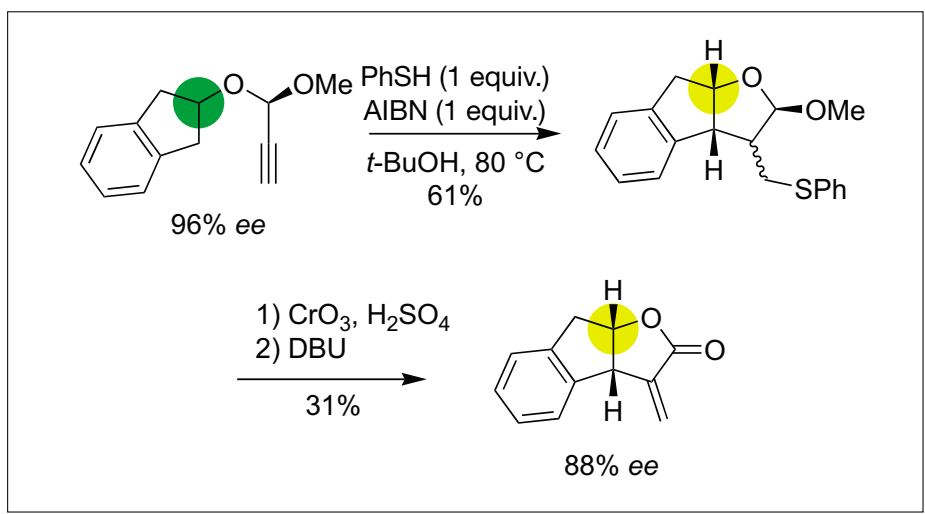

Scheme 10.

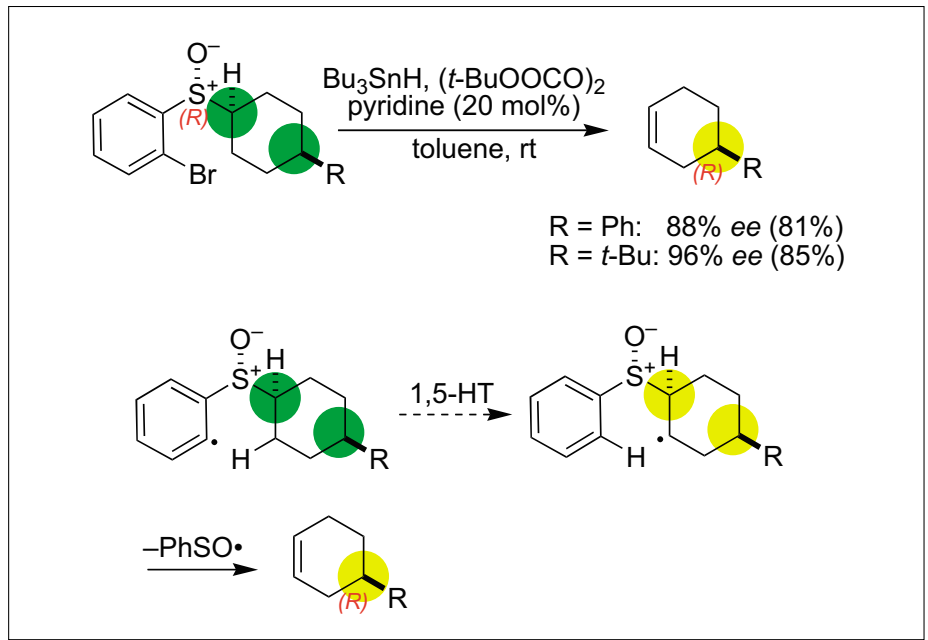

Scheme 11. enantiomeric excess. The diastereoselectivity of the hydrogen atom abstraction step is greater than 95:5.

\section{Translocation-Elimination Processes}

The thermal syn elimination of sulfoxides is a well-known reaction occurring at temperatures higher than $200{ }^{\circ} \mathrm{C}$ when nonstabilized alkenes are formed. This reaction has been applied in a pioneering work of Goldberg and Sahli for the preparation of 4-substituted cyclohexene. ${ }^{[15]}$ We were very interested in finding a mild alternative to this process. ${ }^{[16]}$ We discovered, that aryl radicals generated for ortho-bromophenyl sulfoxides undergoes 1,5-hydrogen atom translocation. The $\beta$-sulfinylated radical fragments extremely rapidly to afford the corresponding alkene. Starting from enantiomerically pure material, 4-substituted cyclohexenes have been prepared with enantiomeric excesses up to $96 \%$ ee (Scheme 11) indicating that the radical translocation process is taking place with a high level of stereoselectivity. The reaction conditions described in the 
preliminary communication ${ }^{[16]}$ are leading to some racemization of the sulfoxide before elimination. The racemization is not observed when using the reaction conditions described in Scheme $11\left(\mathrm{Bu}_{3} \mathrm{SnH}\right.$, ditert-butyl peroxalate, pyridine, in toluene at room temperature) and higher enantioselectivities are thus obtained. ${ }^{[17]}$

A model for the transition state of the reaction supported by $a b$ initio calculations (UHF 6-31G*) is reported in the Fig. The cyclohexane ring lies in a chair conformation, and the sulfinyl group occupies an axial position. The preferred transition state minimizes the steric interactions between the oxygen atom of the sulfoxide and the cyclohexyl group. The minor transition state is destabilized by interaction of the oxygen atom with the cyclohexyl ring.

\section{Conclusions}

The stereoselectivity of intramolecular 1,5-hydrogen transfers varies from poor to excellent depending on the substrates and the reaction conditions such as the reaction temperature. Transition state models based on steric interactions and stereoelectronic effects allow rationalization of the stereochemical outcome of these processes. Radical translocations represents a unique mode to activate remote $\mathrm{C}-\mathrm{H}$ bonds and the stereochemical control of such process reinforce their synthetic potential, particularly for application in target oriented synthesis such as natural product synthesis.

Received: May 9, 2008

[1] For general reviews on radical reactions, see: B. Giese in 'Radicals in Organics Synthesis: Formation of Carbon-Carbon Bonds', Pergamon, Oxford, 1988; D. P. Curran in 'Comprehensive Organic Synthesis', Ed. B. M. Trost, I. Fleming, M. F. Semmelhack, Pergamon, Oxford, 1991, Vol. 4, pp 715 and 779; W. B. Motherwell, D. Crich in 'Free Radical

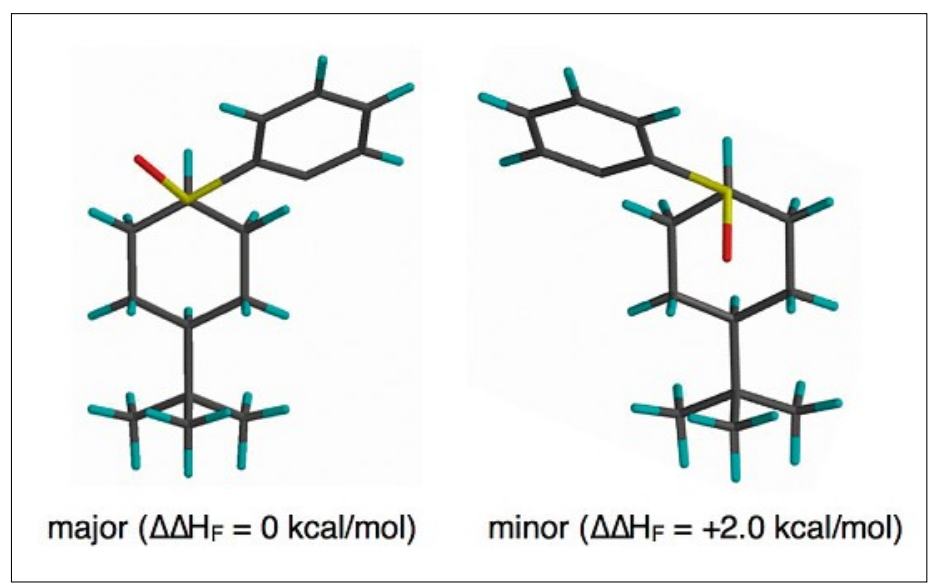

Fig.

Chain Reactions in Organic Synthesis', Academic Press, London, 1992; J. Fossey, D. Lefort, J. Sorba in 'Free Radicals in Organic Synthesis', Wiley, Chichester, 1995; 'Radicals in Organic Synthesis', Ed. P. Renaud, M. P. Sibi, Wiley-VCH, Weinheim, 2001; S. Zard, 'Radical Reactions in Organic Synthesis', Oxford University Press: Oxford, 2003; 'Radicals in Synthesis I and II' (Topic in Current Chemistry 263 and 264), Ed. A. Gansäuer, Springer, Berlin, 2006.

[2] A. L. J. Beckwith, C. H. Schiesser, Tetrahedron 1985, 41, 3925; D. C. Spellmeyer, K. N. Houk, J. Org. Chem. 1987, 52, 959.

[3] L. Feray, N. Kouznetsov, P. Renaud, in 'Radicals in Organic Synthesis', Vol. 2, Ed. P. Renaud, M. P. Sibi, Wiley-VCH, Weinheim, 2001, pp. 246; J. Robertson, J. Pillai, R. K. Lush, Chem. Soc. Rev. 2001, 30, 94; Z. Cekovic, J. Serb. Chem. Soc. 2005, 70, 287.

[4] M. S. Chen, M. C. White, Science 2007, 318, 783.

[5] T. Katsuki, in 'Radicals in Organic Synthesis', Vol. 2, Ed, P. Renaud, M. P. Sibi, Wiley-VCH, Weinheim, 2001, pp. 113.

[6] M. Gulea, J. M. Lopez-Romero, L. Fensterbank, M. Malacria, Org. Lett. 2000, 2, 2591.
[7] G. Petrovic, R. N. Saicic, Z. Cekovic, Tetrahedron Lett. 1997, 38, 7107.

[8] G. Petrovic, Z. Cekovic, Tetrahedron 1999, 55, 1377.

[9] I. Ryu, Chem. Soc. Rev. 2001, 30, 16.

[10] S. Tsunoi, I. Ryu, T. Okuda, M. Tanaka, M. Komatsu, N. Sonoda, J. Am. Chem. Soc. 1998, 120, 8692.

[11] L. R. Reddy, B. V. Reddy, E. J. Corey, Org. Lett. 2006, 8, 2819.

[12] S. Bogen, M. Gulea, L. Fensterbank, M. Malacria, J. Org. Chem. 1999, 64, 4920.

[13] P. Renaud, F. Beaufils, L. Feray, K. Schenk, Angew. Chem. Int. Ed. 2003, 42 , 4230.

[14] F. Villar, O. Equey, T. Kolly-Kovac, P. Renaud, Chem. Eur. J. 2003, 9, 1566; O. Corminboeuf, P. Renaud, C. H. Schiesser, Chem. Eur. J. 2003, 9, 1578.

[15] S. I. Goldberg, M. S. Sahli, J. Org. Chem. 1967, 32, 2059; S. I. Goldberg, M. S. Sahli, Tetrahedron Lett. 1965, 4441.

[16] C. Imboden, F. Villar, P. Renaud, Org. Lett. 1999, 1, 873.

[17] N. Kuznetsov, P. Renaud, publication in preparation. 